\title{
Droplet size distribution in homogeneous isotropic turbulence
}

\author{
Prasad Perlekar, ${ }^{1}$ Luca Biferale, ${ }^{2}$ Mauro Sbragaglia, ${ }^{2}$ Sudhir Srivastava, ${ }^{3}$ and Federico Toschi ${ }^{4}$ \\ 1) International Collaboration for Turbulence Research, Dept. Physics and Dept. Mathematics $E_{3}$ Computer \\ Science and J. M. Burgers Centre for Fluid Dynamics, \\ Eindhoven University of Technology, 5600 MB Eindhoven, The Netherlands. ${ }^{\text {a) }}$ \\ ${ }^{2)}$ International Collaboration for Turbulence Research, Dept. Physics and INFN, \\ University of Rome "Tor Vergata" Via della Ricerca Scientifica 1, 00133 Rome, \\ Italy. \\ 3) Dept. Physics and Dept. Mathematics \& Computer Science and J. M. Burgers Centre for Fluid \\ Dynamics, \\ Eindhoven University of Technology, 5600 MB Eindhoven, The Netherlands. \\ ${ }^{4)}$ International Collaboration for Turbulence Research, Dept. Physics and Dept. Mathematics ${ }_{3}$ Computer \\ Science and J. M. Burgers Centre for Fluid Dynamics, \\ Eindhoven University of Technology, 5600 MB Eindhoven, The Netherlands and \\ CNR-IAC, Via dei Taurini 19, 00185 Rome, Italy.
}

(Dated: 12 November 2018)

We study the physics of droplet breakup in a statistically stationary homogeneous and isotropic turbulent flow by means of high resolution numerical investigations based on the multicomponent lattice Boltzmann method. We verified the validity of the criterion proposed by Hinze (1955) for droplet breakup and we measured the full probability distribution function (pdf) of droplets radii at different Reynolds numbers and for different volume fraction. By means of a Lagrangian tracking we could follow individual droplets along their trajectories, define a local Weber number based on the velocity gradients and study its cross-correlation with droplet deformation.

\section{INTRODUCTION}

Droplets in turbulent flows occur in variety of industrial processes such as sprays, colloid mills, and mixers. When the droplet diameter are smaller than the dissipative scales of turbulence, one can approximate them as point particles whose dynamics is governed by the Maxey-Riley-Gatignol equations ${ }^{1,2}$. Several numerical, analytical, and experimental studies have studied this regime and found interesting phenomena such as clustering of particles at small and large scales ${ }^{3,4}$. When the diameter of the droplet is larger than the dissipative scales of turbulence, the point particle approximation is no longer valid. Inertial scale sized droplets deform, break, and coalesce under the action of turbulence. The degree of deformation is governed by the ratio of the surface tension forces and the intensity of turbulence or the Weber number, $W e$ :

$$
W e \equiv \frac{\rho^{(m)}\left\langle\left(\delta u_{D}\right)^{2}\right\rangle D}{\sigma}
$$

where $\rho^{(m)}$ is the density of the carrier medium fluid, $\delta u_{D}$ is the average velocity difference across the droplet, the angular bracket indicate spatial averaging, $D$ the droplet diameter, and $\sigma$ the surface tension. If inertia forces overwhelm the surface tension $W e>1$, droplet breaks. Using Kolmogorov theory for velocity differences $\left\langle\left(\delta u_{D}\right)^{2}\right\rangle \sim \varepsilon^{2 / 3} D^{2 / 3}$ where $\varepsilon$ is the energy dissipation rate, Hinze showed, in his 1955 seminal work ${ }^{5}$, that the maximum droplet diameter that does not undergo breakup is given by criterion:

$$
D_{\max }=C\left(\frac{\rho^{(m)}}{\sigma}\right)^{-3 / 5} \varepsilon^{-2 / 5}
$$

where the coefficient $C=0.725$ is obtained by fitting with experimental data. The above argument does not take into account the coagulation of droplets which constitute an important mechanism in dense suspensions. Even in dilute limit because of droplet breakup and collision events one expects the droplet dispersion pdf to have a finite width peaked around $D_{\max }$. Breaking rate is strongly correlated with the underlying turbulent stress coarse grained on a scale of the size of droplet. The latter quantity in fully developed turbulence is

a) Corresponding author:p.perlekar@tue.nl 
strongly non-Gaussian, with maximal deviations from Gussianity observed in the viscous-inertial intermediate range. Understanding the stationary droplet distribution due to break-up and coagulation at changing Reynolds numbers and droplet volume fraction remains a key unsolved problem.

Experimental studies by Pacek et al. ${ }^{6,7}$ focused on turbulent dispersions where the carrier medium and the droplet phase have the same density and viscosity and observed droplets size distributions consistent with log-normal. Andersson et al. ${ }^{8}$ studied both bubble and droplet breakup experimentally and found that single bubbles primarily undergoes binary breakups while droplets typically present multiple breakups. Risso and Fabre studied the oscillations and breakup of bubbles in turbulent flow ${ }^{9}$ while Ravelet et al. ${ }^{10}$ focused on breakups of a bubbles in turbulence rising due to gravity. The experimental studies of Eastwood et $a l .{ }^{11}$ focused on the breakup of bubbles in turbulent jets and reported deviations from what predicted by the Hinze criterion ${ }^{5}$.

To date few numerical studies exist, this is probably due to the additional difficulties implied with the need for interface tracking under highly turbulent conditions. Further, to reach good statistical convergence, very long and computationally expensive stationary runs are needed. Numerical works by Qian et al. ${ }^{12}$ studied first few breakup events in a high-density contrast bubble breakup and were able to reproduce the experimental results of Fabre et al. ${ }^{9}$. Derksen et al. ${ }^{13}$ studied how turbulence history can effect coarsening by switching off turbulence in a droplet dispersion.

The aim of the present paper is to understand the interplay between turbulent fluctuations and surface tension in a turbulent emulsion. To this end, we always keep densities and viscosities of the droplet and of the medium identical $\left(\rho^{(d)} / \rho^{(m)}=1\right.$ and $\left.\nu^{(d)} / \nu^{(m)}=1\right)$. We focus on cases with different droplet volume fractions $\phi=V_{d} / V_{m}$, where $V_{d}$ and $V_{m}$ design volumes of the droplet or of the carrier phase respectively. Furthermore, to avoid any effects of boundaries, we limit to homogeneous and isotropic turbulence. Our main results are: (a) we show that a stationary droplet emulsion can be maintained for arbitrary long times, with a chosen turbulence intensity and volume fraction, $\phi ;(\mathrm{b})$ the average droplet diameter is in agreement with what predicted by the Hinze criterion at least for small values of $\phi$; (c) droplet pdf show peaks close to the average droplet radius and broadens at increasing $\phi ;$ (d) by means of Lagrangian tracking of individual droplets trajectory we show that breakup events are typically associated with peaks in the local energy dissipation rate in the droplet neighborhood.

\section{NUMERICAL METHOD}

We model the droplets and the carrier fluid phase by means of a multicomponent Lattice-Boltzmann (LBM) algorithm with, by now standard, non-ideal interactions as introduced by Shan-Chen ${ }^{14-16}$. This is a well established numerical method thus we provide here only its key details. The stirring mechanism, which is needed in order to keep the system in a stationary turbulent state, is applied via a large-scale forcing.

The lattice Boltzmann equations for the Shan-Chen multicomponent D3Q19 model are:

$$
\begin{array}{r}
f_{i}^{(\alpha)}\left(\boldsymbol{x}+\boldsymbol{e}_{i}, t+1\right)=f_{i}^{(\alpha)}(\boldsymbol{x})-\frac{1}{\tau_{\alpha}}\left[f_{i}^{(\alpha)}(\boldsymbol{x}, t)-\bar{f}_{i}^{(\alpha)}(\rho, \boldsymbol{u})\right] \\
\bar{f}_{i}^{(\alpha)}(\rho, \boldsymbol{u})=\rho^{(\alpha)} w_{i}\left[1+\frac{\boldsymbol{e}_{i} \cdot \boldsymbol{u}}{c_{s}^{2}}+\frac{\boldsymbol{u} \boldsymbol{u}:\left(\boldsymbol{e}_{i} \boldsymbol{e}_{i}-c_{s}^{2} \boldsymbol{I}\right)}{2 c_{s}^{4}}\right] \\
\rho^{(\alpha)}=\sum_{i} f_{i}^{(\alpha)} ; \boldsymbol{u}^{(\alpha)}(\boldsymbol{x}, t)=\sum_{i} \boldsymbol{e}_{i} f_{i}^{(\alpha)}(\boldsymbol{x}, t) .
\end{array}
$$

Here $f_{i}^{\alpha}(\boldsymbol{x}, t)$ are the LBM distribution function at position $\boldsymbol{x}$ and time $t$ for the fluid component $\alpha=\{d, m\}$ (droplet and medium, respectively). The fluid densities and velocities of the individual components are $\rho^{(\alpha)}$ and $\boldsymbol{u}^{(\alpha)}$. Here $w_{i}, \boldsymbol{e}_{i}$ are the LBM weights and lattice speeds, $i=\{0, \ldots, 18\}^{17,18}$. The total density of the fluid is the sum of the density of the two components $\rho=\sum_{\alpha} \rho^{(\alpha)}$ while the total hydrodynamic velocity is $\boldsymbol{u}=\sum_{\alpha} u^{(\alpha)} \rho^{(\alpha)} / \rho$. The global effective kinematic viscosity of the fluid is related to the relaxation times of its components $\nu=\sum_{\alpha} c_{s}^{2}\left(\tau^{(\alpha)} c^{(\alpha)}-0.5\right)^{16}, c^{(\alpha)}=\rho^{(\alpha)} / \rho$ is the concentration, and finally $c_{s}=1 / \sqrt{3}$ is the speed of sound on the lattice for the D3Q19.

The non-ideal nature of the fluid is introduced by adding an extra force to the LBM equilibrium velocity 
TABLE I. Runs parameters. For runs N128A $-128 \mathrm{D}$ and N512A, $\rho^{(m)}+\rho^{(d)}=2.4$. For run N512B, $\rho^{(m)}+\rho^{(d)}=1.077$. Here $\nu$ is the viscosity of the multicomponent fluid, $G$ is the interaction strength and $R_{\lambda}$ is the Taylor's based Reynolds number ${ }^{21}$. The relaxation time of the two fluids $\tau=0.515$ was kept fixed.

\begin{tabular}{lccccccc}
\hline \hline & $N$ & $G$ & $\varepsilon$ & $R e_{\lambda}$ & $\nu$ & $\sigma$ & $\phi($ in $\%)$ \\
\hline \hline N128A & 128 & 0.3 & $4.0 \cdot 10^{-8}$ & 15 & $5 \cdot 10^{-3}$ & $1.6 \cdot 10^{-3}$ & 0.07 \\
N128B & 128 & 0.3 & $4.0 \cdot 10^{-8}$ & 15 & $5 \cdot 10^{-3}$ & $1.6 \cdot 10^{-3}$ & 0.5 \\
N128C & 128 & 0.3 & $4.0 \cdot 10^{-8}$ & 15 & $5 \cdot 10^{-3}$ & $1.6 \cdot 10^{-3}$ & 5.0 \\
N128D & 128 & 0.3 & $4.0 \cdot 10^{-8}$ & 15 & $5 \cdot 10^{-3}$ & $1.6 \cdot 10^{-3}$ & 10 \\
N512A & 512 & 0.3 & $2.9 \cdot 10^{-9}$ & 30 & $5 \cdot 10^{-3}$ & $1.6 \cdot 10^{-3}$ & 0.3 \\
N512B & 512 & 0.1 & $2.9 \cdot 10^{-9}$ & 30 & $5 \cdot 10^{-3}$ & $1.7 \cdot 10^{-3}$ & 0.3 \\
\hline \hline
\end{tabular}

$\operatorname{as}^{15}$ :

$$
\overline{\boldsymbol{u}}^{(\alpha)}=\boldsymbol{u}^{\prime}+\frac{\tau^{(\alpha)} \boldsymbol{F}_{S C}^{(\alpha)}}{\rho^{(\alpha)}} \text { and } \boldsymbol{u}^{\prime}=\frac{\sum_{\alpha} \rho^{(\alpha)} \boldsymbol{u}^{(\alpha)} / \tau^{(\alpha)}}{\sum_{\alpha} \rho^{(\alpha)} / \tau^{(\alpha)}}
$$

The non-ideal interaction, as proposed by Shan and Chen, is ${ }^{15}$ :

$$
\mathbf{F}_{S C}^{(\alpha)}=-G \rho^{(\alpha)}(\boldsymbol{x}) \sum_{i, \alpha \neq \alpha^{\prime}} \rho^{\left(\alpha^{\prime}\right)}\left(\boldsymbol{x}+\boldsymbol{e}_{i}\right) w_{i} \boldsymbol{e}_{i}
$$

where $\left\{\alpha, \alpha^{\prime}\right\}=\{d, m\}$ and the coupling parameter, $G$, determines the strength of the microscopic interaction and effectively sets the value of the surface tension and the diffusivity ${ }^{19}$. Under appropriate conditions this force allows the formation of interface between the different fluid components.

As already anticipated in this study we limit ourself to the case $\tau^{(d)}=\tau^{(m)}=\tau$ which implies $\nu^{(d)}=$ $\nu^{(m)}=\nu$, i.e. identical viscosities for the two phases. The total fluid density $\rho^{(d)}+\rho^{(m)}$ can be considered as constant over the entire domain, except for the small variations at the droplet interface. This approach to model turbulent emulsion has the advantage of being simple and computationally efficient. It was however noticed that for extremely long simulation times, as those needed in order to collect firm statistical information, one can observe important diffusion of one fluid component into the other. This physical phenomenon can be artificially large in the LBM simulations due to the relatively big interface width between the two fluid components. We have however shown in a recent publication ${ }^{20}$ how this effect can be controlled by means of an effective and computationally efficient manner. By means of this improvement, the presented algorithm allows us to investigate turbulent emulsions for arbitrarily long simulations times.

In order to stir turbulence we apply forcing at each position and at each time step modulated by means of a sum of sine waves with small wavenumbers. To ensure an homogeneous and isotropic stirring, the phases of the sine waves are evolved in time by means of a stochastic process. The forcing is divergenceless and its expression for the generic $i$-th component is:

$$
F_{i}^{(\alpha)}(\boldsymbol{x}, t)=A \rho^{(\alpha)} \sum_{j \neq i}\left[\sin \left(k_{j} x_{j}+\Phi_{k}^{(j)}(t)\right)\right]
$$

where $i, j=\{1,2,3\}, \mathbf{F}^{(\alpha)}$ is the external force at $\boldsymbol{x}$ and time $t . A$ controls the forcing amplitude, $k_{j}$ are the wave-vector components and the sum is limited to $k^{2}=k_{1}^{2}+k_{2}^{2}+k_{3}^{2} \leq 2$. The phases $\Phi_{k}^{(j)}$ are evolved in time according to independent Ornstein-Uhlenbeck processes with the same relaxation times $T=u_{r m s} / N$ ( $N$ is the linear domain size and $u_{r m s}$ was taken equal to 0.1 to almost match with the typical values for the large scale velocity).

\section{THE STATIONARY STATE}

In presence of turbulence droplets continuously undergo breakup and collision processes, whose rate depends on both volume fraction and Reynolds number. The plot in Fig. 1 shows a typical time evolution of the droplet number for volume fractions $\phi$ equal to $0.5 \%, 5 \%$ and $10 \%$ at $R e_{\lambda}=15$ (runs N128B - D, Table $I$ ). 

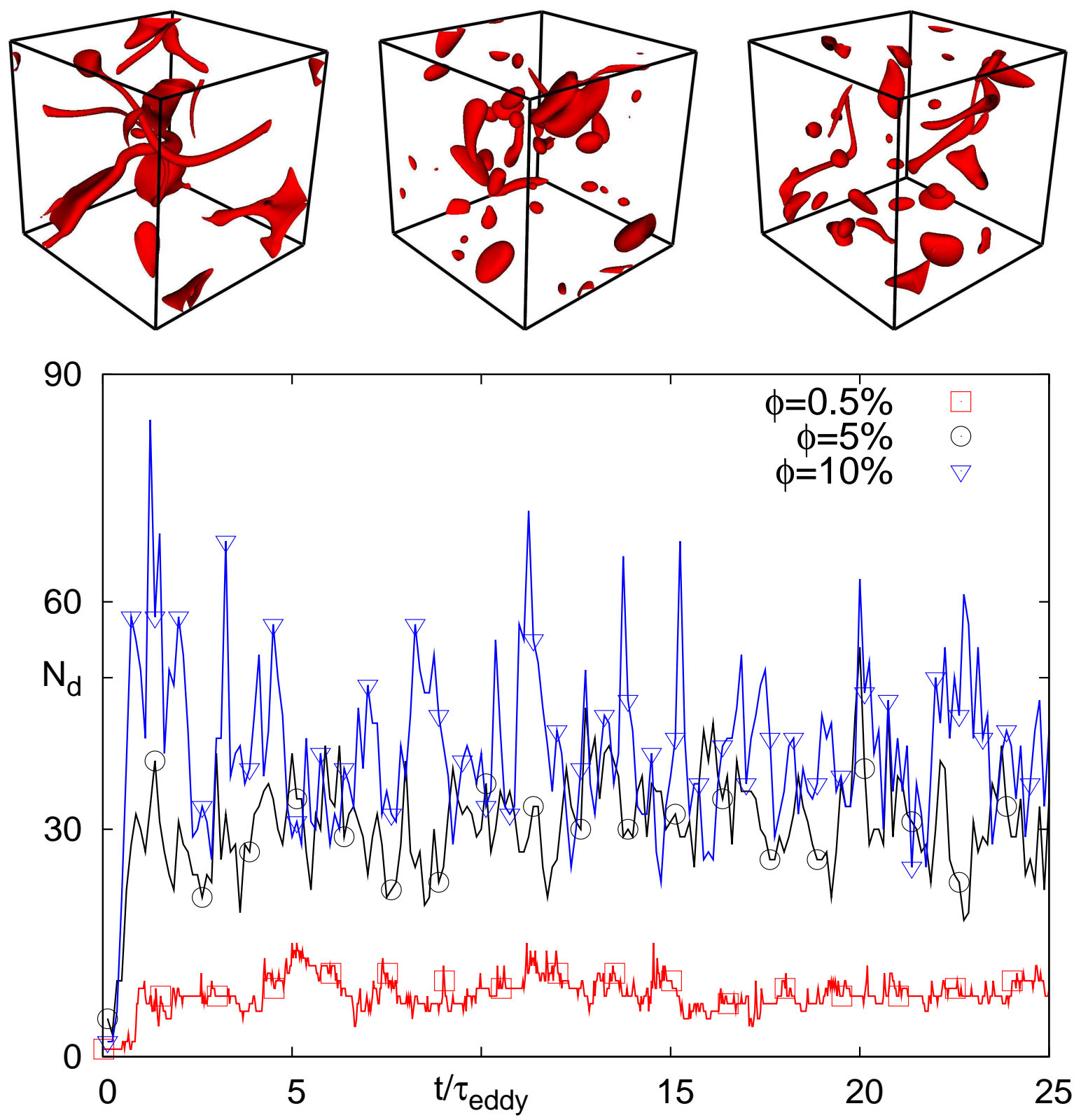

FIG. 1. Number of droplets vs. time for different values of the volume fraction, $\phi$. An increase in the volume fraction leads to an increase in the total droplets number and more coagulation processes occur. The time is made dimensionless by the large scale eddy turnover time, $\tau_{e d d y}=N / u_{r m s}$. Top panel shows snapshots of the droplet dispersion at the intermediate $t / \tau_{\text {eddy }}=0.33$ and steady state $t / \tau_{\text {eddy }}=12.5,25$ configurations.

During the early simulation stages, when turbulence is still developing, the droplet deforms and then starts to breaks up into smaller droplets. At later times, the droplet count steadily oscillates around a mean value because of a continuos competition between breakups and coalescence events. The stationary probability distribution function of droplet sizes, with its corresponding cumulative distribution, is shown in Fig. 2 for different volume fractions $\phi=0.07 \%, \phi=0.5 \%$ and $\phi=5 \%$ with $R e_{\lambda}=15$ and $\sigma=1.6 \cdot 10^{-3}$ (runs N128A - C, Table I).

In the case $\phi=0.07 \%$ only very few breakups are observed and the droplet PDF closely resemble a delta function. We observe a mild increase in the average droplet radius at increasing the volume fraction of the droplet phase; furthermore, because of enhanced coalescence, we observe that the maximum droplet radius increases with increasing the volume fraction (see Fig. 2(bottom)). In the same figure we also superpose the empirical pdf with a log-normal distribution. 
As one can see, the two fits are in good agreement with the data, within statistical errors, at large and intermediate radiuses. At very small droplet radius one can observe deviations between measured data and the fitting function. On this point we are not able to draw conclusive statement and we tend to think that deviations may be unphysical and due to the limitations of the diffused interface models to capture droplets whose size is comparable to the interface width.
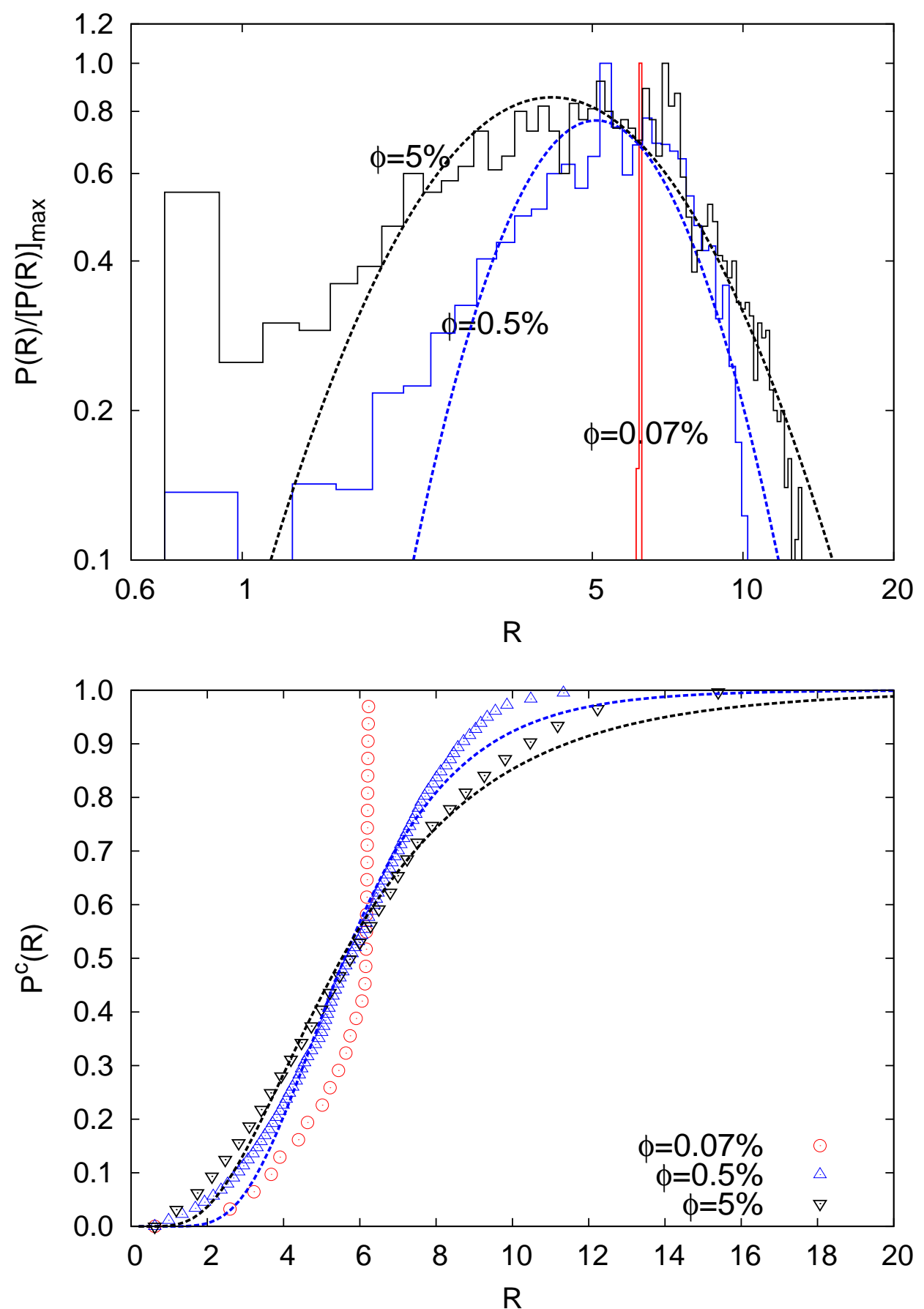

FIG. 2. (top-panel) Log-log plot of the probability distribution function (pdf) of droplet radius for different values of the volume fraction. Pdf are scaled to have maximum value unity. (bottom-panel) Cumulative probability distribution function for different volume fractions. In panels, dashed lines represent log-normal fits to distributions with $\phi=0.5 \%$ and $\phi=5 \%$. 


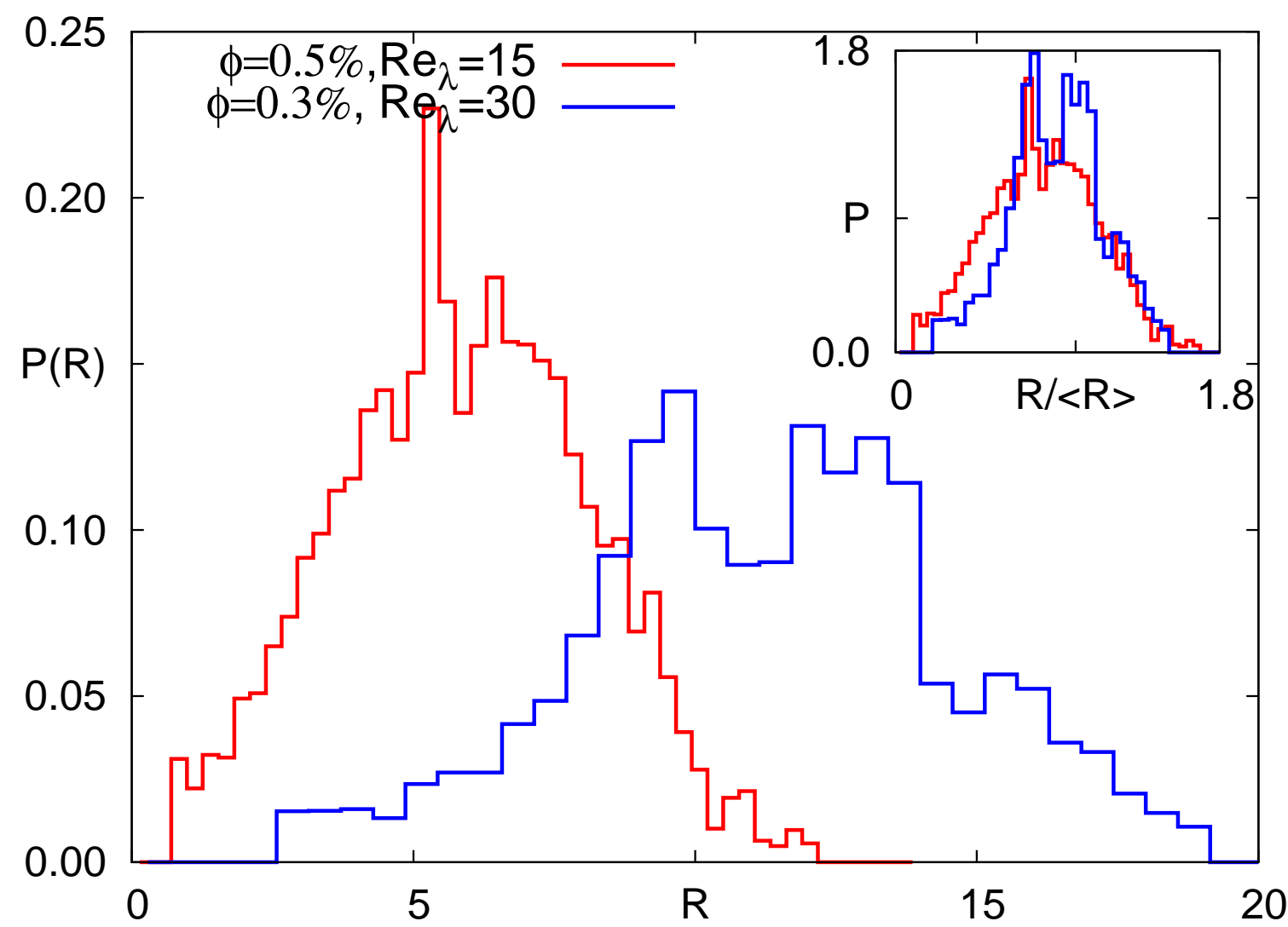

FIG. 3. Probability distribution function of the droplet radius from two simulations with almost the same volume fraction but with different $R e_{\lambda}$. The inset shows the pdf normalized by the average droplet radius.

To understand the effect of the Reynolds number on the stationary droplet size distribution, in Fig. 3 we compare the droplet radius PDFs with comparable volume fractions but for different Reynolds number $\phi=0.5 \%, R e_{\lambda}=15$ and $\phi=0.3 \%, R e_{\lambda}=30$ (corresponding to runs N128B and N512A, Table I). Our result support the validity of the Hinze criterion which predicts that, for the same material parameters, the average droplet diameter should only depend inversely on the energy dissipation rate [Eq. (2)]. By rescaling the droplet radius with their average values, the two PDF collapse (inset of Fig. 3). Thus, we can exclude strong dependency of the pdf shape, at fixed volume fraction and material properties, on the Reynolds number; at least this is the case for the two Reynolds number that we investigated.

\section{HINZE CRITERION}

The Hinze criterion, as in Eq. (2), provides the estimate for the maximum droplet diameter, $D_{\max }$, that should not undergo breakup at a fixed turbulence intensity. In presence of turbulence however breakup and coalescence always occurs and the correct indicator for the droplet size is the average droplet radius. We replace therefore $D_{\max }$ by the average droplet diameter $\langle D\rangle$, where with the angular bracket we indicate averaging over a steady distribution of droplet diameters. From Fig. 4 one can see that our data support the validity of Hinze criterion in the case of small volume fractions, while we one can clearly detect a non trivial dependency on the volume fraction for larger values of $\phi$ (keeping the turbulent intensity and surface tension constant).

As the droplet is transported by the turbulent flow, it visits regions of where velocity gradients are much larger or smaller than the average value. Thus, a better characterization of the droplet breakup required the tracking of individual droplets along their trajectory along with monitoring their deformation and the local flow conditions in the immediate neighboroud, i.e. the local Weber number, $W e_{s}$. Here we define the local 


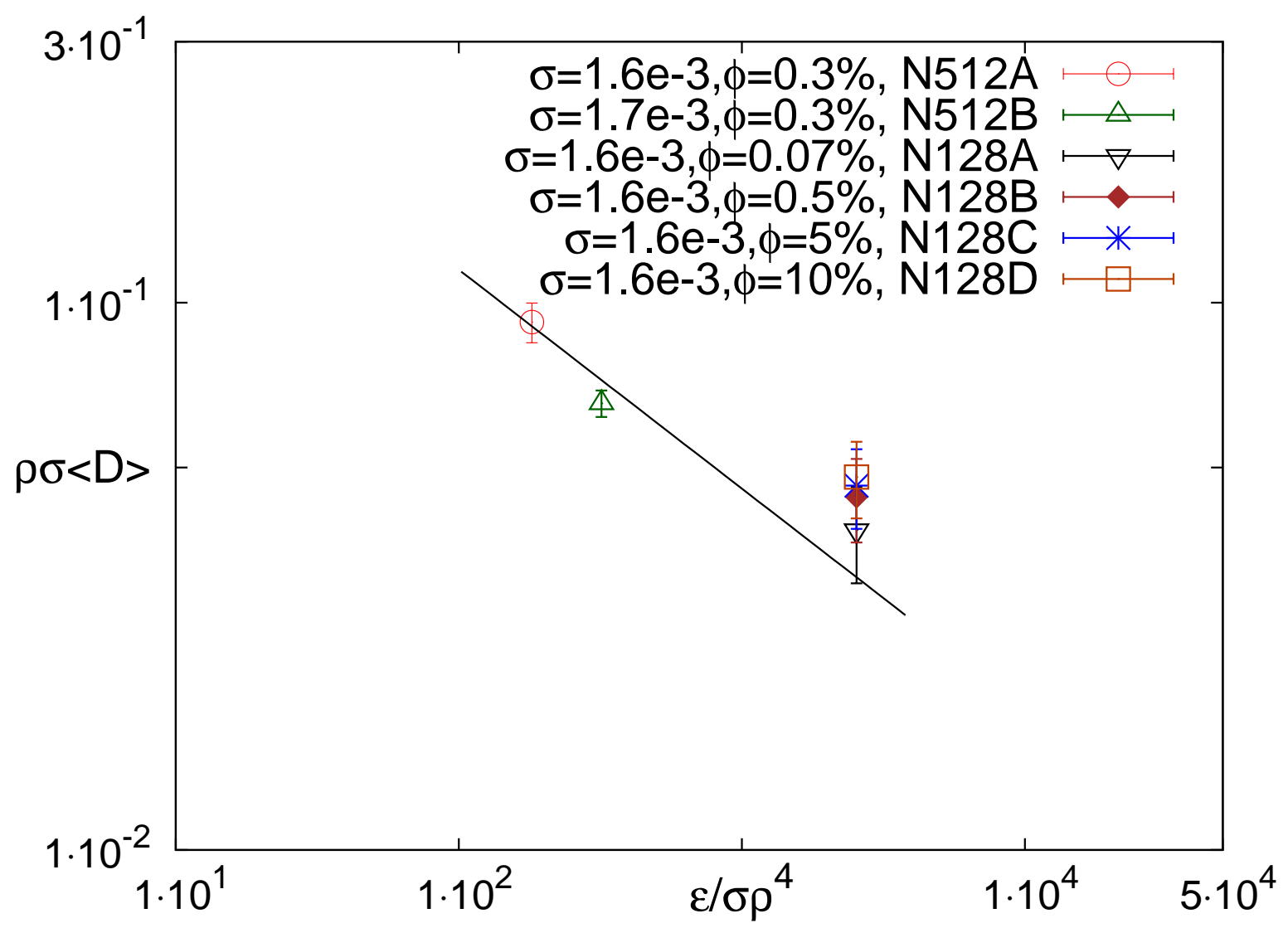

FIG. 4. Droplet diameter versus the energy dissipation rate to test the Hinze criterion for droplet breakup ${ }^{5}$. The different symbols correspond to runs that are distinguished by their volume fraction $\phi$ and surface tension $\sigma$ (see Table I). The solid black line is the prediction of Hinze [Eq. (2)]. Note how at larger volume fractions deviations from the Hinze criterion are observed, these are probably due to enhancement of coagulation events. The error-bars indicate the uncertainty in the estimation of the droplet interface position.

Weber number as the difference between the maximum velocity difference between a point on the droplet surface and the velocity of the droplet center of mass $W e_{s} \equiv\left|\boldsymbol{u}_{\max , S}-\boldsymbol{u}_{C M}\right|$. The droplet deformation is instead characterized by a dimensionless parameter $S^{*}=\left(S / S_{0}-1\right)$ where $S$ is the surface area of the droplet and $S_{0} \equiv(4 \pi)^{1 / 3}\left(3 V_{d}\right)^{2 / 3}$ is the surface area of the equivalent spherical droplet (i.e. a spherical droplet with the same volume of the deformed one). With this definition $S^{*}=0$ corresponds to an (undeformed, i.e. spherical) droplet, whereas $S^{*}>0$ indicate a stretched droplet. The plot in Fig. 5 shows the time evolution of the surface deformation, $S^{*}$, of the local Weber number $W e_{s}$, and times at which breakup event occur, during a part of the droplet trajectory. Data in Fig. 5 refers to the run with the smallest volume fraction, $\phi=0.07$, and with $R e_{\lambda}=15$. For this run, due to its low droplet count, it was technically easier to track breakup events (run N128A, Table I). As one can see, the droplet deformation, $S^{*}$, correlates strongly with the local Weber number, $W e_{s}$. Furthermore, just before the droplet breaks (stars in the figure) one can identify a sharp increase in both surface deformation $S^{*}$ and $W e_{s}$. We can further quantify the correlation by computing the joint pdf of $S^{*}$ and $W e_{s}$ over the full time-series for run N128A (see Fig. 6). From this joint $\mathrm{PDF}$ we find that $S^{*} \approx 0.066 \mathrm{We}$. In Ref. ${ }^{12}$ it was reported a similar correlation but for the rather different case of a vapor-liquid dispersion with a density contrast between the two phases.

\section{v. CONCLUSIONS}

We have studied the droplet dispersion in a turbulent emulsion from very small to larger volume fractions. We focused on the role of turbulence fluctuations on the droplet deformation and breakup and to this end we limited to density and viscosity matched emulsions. In this way we could better highlight the new physics associated with the interplay between surface tension and turbulence fluctuations. Our numerical study, 


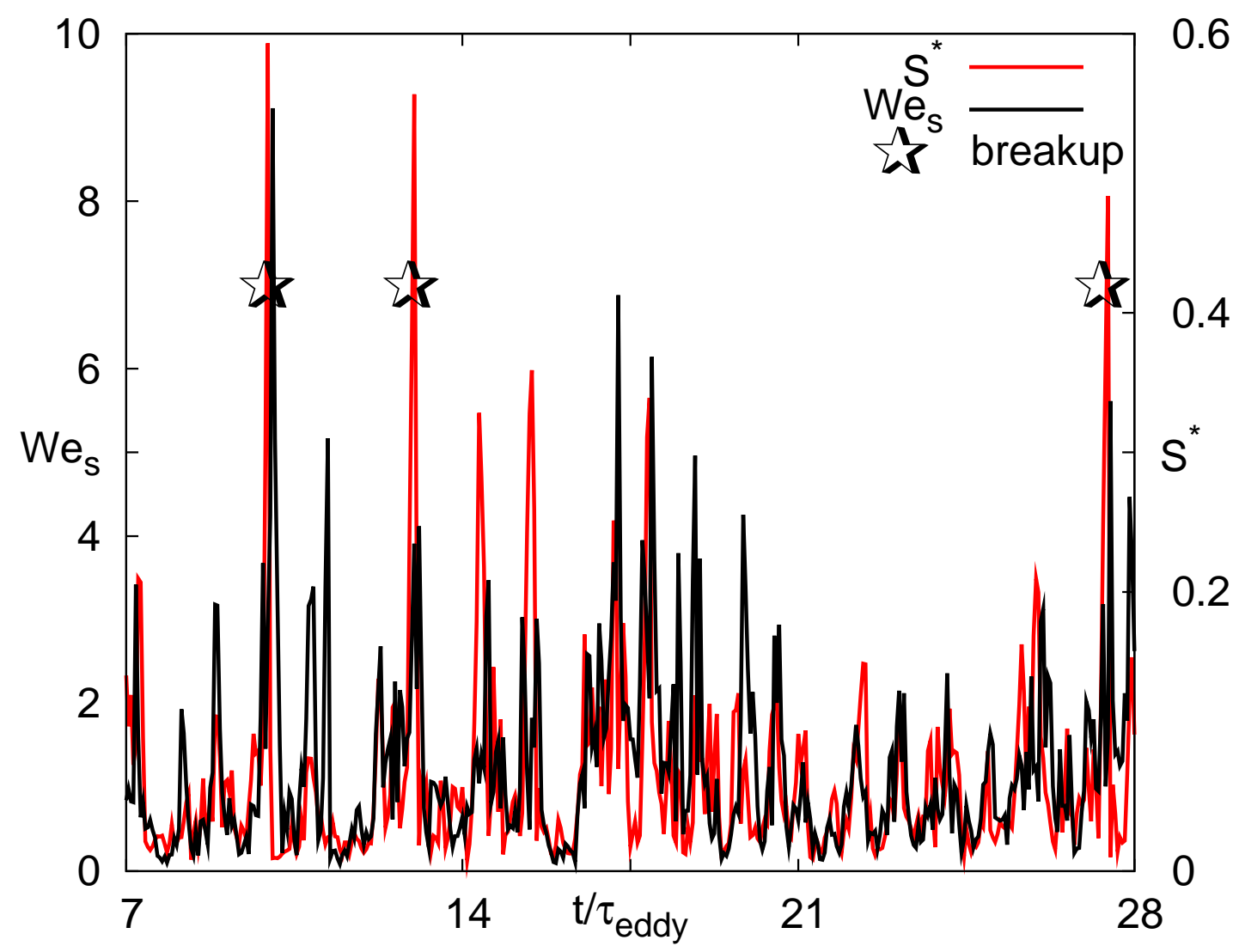

FIG. 5. Correlation between the Weber number and the non-dimensional droplet extension $S^{*} \equiv\left(S / S_{0}-1\right)$. The instantaneous Weber number is computed by using the maximum value of the energy dissipation at the droplet surface $W e_{s}$. The plot represent the evolution of a typical droplet trajectory from the run with smallest volume fraction $\phi=0.07 \%$ and $R e_{\lambda}=15(\mathrm{~N} 128 \mathrm{~A})$. Stars indicate the breakup events that we observed during this particular run.

which is based on the lattice Boltzmann method, shows that by using a proper large-scale isotropic stirring mechanism ${ }^{20}$, it is possible to attain a stationary steady state which allows to study the physics of droplets distribution and evolution in turbulence. The pdf of droplets radii follow a log-normal distribution and the Hinze criterion is well satisfied at small volume fraction, while we observe a departure from its prediction at higher volume fractions. We performed a Lagrangian tracking of individual droplets and we showed that the local Weber number is a suitable prognostic indicator for droplet breakup and correlates strongly with droplet deformation.

\section{ACKNOWLEDGEMENTS}

We thank S. Sundaresan, J. Derksen and H. Xu for discussions. We acknowledge the COST Action MP0806 and FOM (Stichting voor Fundamenteel Onderzoek der Materie) for support. LB, PP, and FT acknowledge the Kavli Institute of Theoretical Physics for hospitality. This research was supported in part by the National Science Foundation under Grant No. NSF PHY05-51164. We acknowledge computational support from CASPUR (Roma, Italy), from CINECA (Bologna, Italy), and from JSC (Juelich, Germany).

${ }^{1}$ M. Maxey and J. Riley, "Equation of motion for a small rigid sphere in a nonuniform flow," Phys. Fluids 26883 (1983).

${ }^{2}$ R. Gatignol, "The faxen formulas for a rigid particle in an unsteady non-uniform stokes flow," J. Mec. Theor. Appl. 1, 143 (1983).

${ }^{3}$ F. Toschi and E. Bodenschatz, "Lagrangian Properties of Particles in Turbulence" Ann. Rev. Fluid Mech. 41, 375 (2009). 


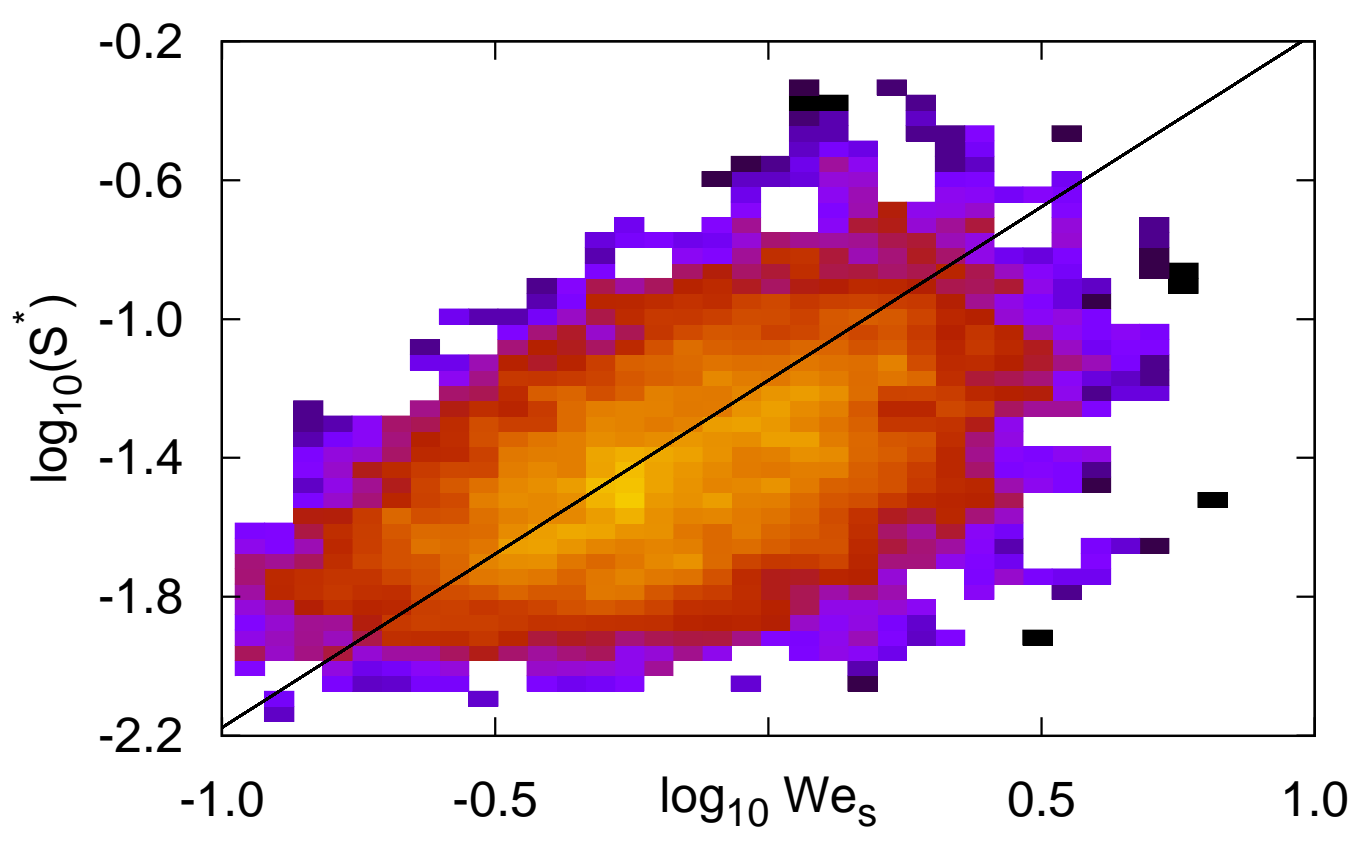

FIG. 6. Log-log plot of the joint probability distribution function of the non-dimensional droplet extension $S^{*}$ versus the local Weber number, as obtained from the velocity at the droplet surface $W e_{s}=\left|u_{\max , S}-u_{C M}\right|$ (see text) for the run with $\phi=0.07 \%$ and $R e_{\lambda}=15$ (N128A). From a least squares fit we obtain $S^{*} \approx 0.066 W e$ (black line) which is in agreement with earlier simulations ${ }^{12}$. In the scatter plot the lighter central region (yellow in color figure) indicate the region of high probability while the darker (black) regions indicate low probability.

${ }^{4}$ J. Bec et al., "Heavy Particle Concentration in Turbulence at Dissipative and Inertial Scales" Phys. Rev. Lett. 98, 084502 (2007).

${ }^{5}$ J.O. Hinze, "Fundamentals of the hydrodynamic mechanism of splitting in dispersion process," A.I.Ch.E. Journal 1, 289 (1955).

${ }^{6}$ A. Pacek, A. Nienow, and I. Moore, "On the structure of turbulent liquid-liquid dispersed flows in an agitated vessel," Chem. Eng. Sci. 49, 3485 (1994).

${ }^{7}$ A. Pacek, C. Man, and A. Nienow, "On the Sauter mean diameter and distributions in turbulent liquid/liquid dispersions in a stirred vessel," Chem. Eng. Sci. 53, 2005 (1998).

${ }^{8}$ R. Andersson and B. Andersson, "On the breakup of fluid particles in turbulent flows," AIChE Journal 52, 2020 (2006).

${ }^{9}$ F. Risso and J. Fabre, "Oscillations and breakup of a bubble in a turbulent field," J. Fluid Mech. 372, 323 (1998).

${ }^{10}$ F. Ravelet, C. Colin, and F. Risso, "On the dynamics and breakup of a bubble rising in a turbulent flow," Phys. Fluids 23, 103301 (2011).

${ }^{11}$ C. Eastwood, L. Armi, and J. Lasheras, "The breakup of immiscible fluids in turbulent flows," J. Fluid Mech 502, 309 (2004).

${ }^{12}$ D. Qian, J. McLaughlin, K. Sankaranarayanan, S. Sundaresan, and K. Kontomaris, "Simulation of bubble breakup dynamics in homogeneous turbulence," Chem. Eng. Comm. 193, 1038 (2006).

${ }^{13}$ J. Derksen and H. V. D. Akker, "Multi-scale simulations of stirred liquid-liquid dispersions," Chem. Eng. Res. 85, 697 (2007).

${ }^{14}$ X. Shan and H. Chen, "Lattice Boltzmann model for simulating flows with multiple phases and components," Phys. Rev. E 47, 1815 (1993).

${ }^{15}$ X. Shan and H. Chen, "Simulation of nonideal gases and liquid-gas phase transitions by the lattice Boltzmann equation," Phys. Rev. E 49, 2941 (1994).

${ }^{16}$ X. Shan and G. Doolen, "Multicomponent Lattice-Boltzmann Model with Interparticle Interaction," J. Stat. Phys. 81, 379 (1995).

${ }^{17}$ S. Succi, The Lattice Boltzmann Equation for Fluid Dynamics and Beyond, (Oxford, Oxford University Press, UK, 2001).

${ }^{18}$ D. A. Wolf-Galdrow, Lattice gas cellular automata and lattice Boltzmann models: an introduction, (Springer, Springer Berlin Heidelberg New York, 2000).

${ }^{19}$ R. Benzi, M. Sbragaglia, S. Succi, M. Bernaschi, and S. Chibbaro, "Mesoscopic lattice Boltzmann modeling of soft-glassy systems: Theory and simulations," J. Chem. Phys. 131, 104903 (2009). 
${ }^{20}$ L. Biferale, P. Perlekar, M. Sbragaglia, S. Srivastava, and F. Toschi, "A Lattice Boltzmann method for turbulent emulsions," J. Phys.: Conf. Ser. (to be published)

${ }^{21}$ U. Frisch, Turbulence the legacy of A.N. Kolmogorov, (Cambridge University Press, Cambridge, 1996). 